\title{
Prevalência de doença renal crônica em adultos no Brasil: revisão sistemática da literatura
}

\author{
Prevalence of chronic renal disease among Brazilian \\ adults: a systematic review
}

\author{
Ana Wanda Guerra Barreto Marinho ${ }^{1,2}$, Anderson da Paz Penha ${ }^{3}$, \\ Marcus Tolentino Silva ${ }^{1,2}$, Taís Freire Galvão ${ }^{2,4}$
}

\begin{abstract}
Resumo
Introdução: A doença renal crônica é um problema global de saúde pública, e no Brasil sua prevalência é incerta. Objetivo: Estimar a prevalência de doença renal crônica em adultos do Brasil. Método: Realizou-se revisão sistemática com buscas no MEDLINE, Embase e outras bases. Dois pesquisadores selecionaram os estudos, extraíram os dados e avaliaram a qualidade. Resultados: Incluímos 16 estudos: inquéritos populacionais que utilizaram critério autorreferido encontraram prevalência nacional de 4,57\% $(1998)$ a 1,43\% (2013); naqueles que usaram hipercreatininemia, a prevalência foi 3,46\% em Bambuí (1997) e 3,13\% em Salvador (2000). Estudos com amostras não representativas usaram critérios clínico-laboratoriais e tiveram maiores prevalências: entre 6,26-7,26\% em campanhas de saúde (2002-2010), 8,94\% em servidores públicos (2008-2010), 9,62\% em usuários de laboratório privado (2003), 27,20\% em pacientes hospitalizados (2013) e 1,35-13,63\% na atenção primária (2010-2012). Pacientes em terapia dialítica representam 0,05\% da população. Discussão: Os estudos com representatividade populacional não aferiram a doença adequadamente, e investigações com melhores critérios diagnósticos tiveram amostragem por conveniência. A heterogeneidade entre pesquisas inviabilizou a elaboração de meta-análise. Conclusão: A prevalência de doença renal crônica variou de acordo com o método empregado na definição da doença. Pelos critérios populacionais, 3-6 milhões teriam a doença. Aproximadamente 100.000 recebem diálise no Brasil.
\end{abstract}

Palavras-chave: falência renal crônica; adulto; prevalência; revisão sistemática; Brasil.

\footnotetext{
Abstract

Introduction: Chronic kidney disease is a global public health issue but its prevalence in Brazil remains uncertain. Objective: To estimate the prevalence of chronic kidney disease among Brazilian adults. Methods: A systematic review through an electronic literature searches on MEDLINE, Embase, other databases plus microdata from national surveys. Two researchers selected, extracted data, and assessed the quality.Results: We included 16 studies: national population-based using self-reported criteria found $4.57 \%$ (1998) to $1.43 \%$ (2013) prevalence; in those employing hypercreatininemia showed rate of 3.46\% in Bambuí (1997) and $3.13 \%$ in Salvador (2000). Studies with non-representative samples employed clinical and laboratory criteria and showed higher prevalences: $6.26 \%$ in campaigns (2010), 8.94\% in public employees (2010), 9.62\% in private laboratory's patients (2003), $27.20 \%$ in hospital (2013), and $1.35-10.64 \%$ in primary care (2011). Patients on dialysis represent $0.05 \%$ of the Brazilian population. Discussion: Representative studies did not adequately assess the disease and investigations with better diagnostic criteria had convenience sampling. Heterogeneity across studies hampered the calculation of meta-analysis. Conclusion: It was not possible

${ }^{1}$ Faculdade de Medicina, Universidade Federal do Amazonas (UFAM) - Manaus (AM), Brasil.

2Programa de Pós-graduação em Ciências Farmacêuticas, Universidade Federal do Amazonas (UFAM) - Manaus (AM), Brasil.

${ }^{3}$ Advanced Studies Program in Clinical Research, University of California - San Diego (CA), Estados Unidos.

${ }^{4}$ Faculdade de Ciências Farmacêuticas, Universidade Estadual de Campinas (Unicamp) - Campinas (SP), Brasil.

Trabalho realizado no município de Manaus (AM), Brasil.

Endereço para correspondência: Ana Wanda Guerra Barreto Marinho - Departamento de Clínica Médica, Faculdade de Medicina, Universidade Federal do Amazonas (UFAM), Rua Afonso Pena, 1053 - Centro - CEP: 69020-160 - Manaus (AM), Brasil - Email: anawandam@gmail.com

Fonte de financiamento: nenhuma.

Conflito de interesses: nada a declarar.
} 
to estimate the prevalence of chronic kidney disease patients in Brazil due to the heterogeneity of studies included in this review. The prevalence varied according to the diagnostic criteria employed among studies. Considering population criteria, 3-6 million people would have the disease. Roughly 100.000 receive dialysis in Brazil.

Keywords: chronic kidney failure, adult, prevalence, review, Brazil.

\section{INTRODUÇÃO}

A doença renal crônica é a perda permanente da função dos rins é reconhecida como um problema global de saúde pública ${ }^{1}$. O aumento no número de casos tem sido reportado na última década em diferentes contextos ${ }^{1-3}$, associados ao envelhecimento e à transição demográfica da população, como resultado da melhora na expectativa de vida e do rápido processo de urbanização $0^{3,4}$. Hipertensão arterial e diabetes são as principais causas ${ }^{5-8}$, ao passo que disparidades socioeconômicas, raciais e de gênero são também fatores determinantes ${ }^{4,9}$.

A detecção precoce e o tratamento adequado em estágios iniciais ajudam a prevenir os desfechos deletérios e a subsequente morbidade relacionados às nefropatias ${ }^{10-12}$. Ademais, resultam em potenciais benefícios para qualidade de vida, longevidade e redução de custos associados ao cuidado em saúde ${ }^{1}$.

A importância da identificação da enfermidade não se restringe somente ao acesso à terapia renal substitutiva ${ }^{1}$. O adequado diagnóstico precoce e tratamento permite reduzir complicações e mortalidade cardiovasculares ${ }^{2-4,13,14}$. Tais metas são desafiadoras onde o acesso aos serviços de saúde é limitado ${ }^{3,6}$, com número reduzido de nefrologistas para o acompanhamento ${ }^{15,16}$.

Em países desenvolvidos, o rastreamento estima prevalência de doença renal crônica entre 10 e $13 \%$ na população adulta ${ }^{17-19}$. Nos países em desenvolvimento, dados de prevalência são limitados e heterogêneos ${ }^{2,13}$. No Brasil, estimativas da prevalência dessa enfermidade são incertas. O conhecimento da prevalência da doença renal crônica entre os brasileiros subsidiaria melhor o planejamento de ações preventivas e assistenciais.

Esta revisão sistemática da literatura teve como objetivo estimar a prevalência de doença renal crônica em adultos no Brasil.

\section{MÉTODOS}

\section{Protocolo e registro}

Trata-se de uma revisão sistemática da literatura, cujo protocolo está registrado na base International Prospective Register of Systematic Reviews (PROSPERO), sob o número CRD42015030054. O estudo utilizou dados secundários provenientes da literatura, prescindindo de aprovação por comitê de ética em pesquisa.

\section{Critérios de elegibilidade}

Incluíram-se os estudos observacionais que estimaram a prevalência de doença renal crônica em adultos no Brasil, definida como a alteração estrutural ou funcional permanente por mais de três meses com implicações à saúde, e classificada de acordo com as causas, taxa de filtração glomerular e albuminúria ${ }^{20}$. Para efeito da presente revisão, consideraram-se adultos os indivíduos com idade $\geq 18$ anos.

\section{Fontes de informação e estratégias de busca}

Pesquisaram-se as bases bibliográficas MEDLINE, Embase, Scopus, LILACS e SciELO, sem restrição de idiomas ou data de publicação. Utilizamos a seguinte estratégia no MEDLINE (via PubMed): ("Renal Insufficiency"[Mesh] OR "Renal Insufficiency, Chronic" [Mesh] OR "Kidney Failure, Chronic"[Mesh] OR "chronic kidney disease" OR "chronic kidney failure" OR CKD OR ESRD OR ESKD OR "end-stage renal disease") AND (“Cross-Sectional Studies" [Mesh] OR "Cross-Sectional”[TIAB] OR "Prevalence" [TIAB] OR "Prevalences"[TIAB] OR "Prevalencia" [TIAB] OR "survey" [TIAB] OR "surveys" [TIAB] OR "population-based"[TIAB]) AND (Brazil OR Brasil). Adaptamos essa estratégia nas demais fontes. A última atualização ocorreu em setembro de 2017.

Também incluímos microdados dos inquéritos nacionais realizados pelo Instituto Brasileiro de Geografia e Estatística (IBGE). Nesses casos, consideramos apenas os questionários respondidos pelos próprios entrevistados, ou seja, excluímos os proxies-respondentes.

Pesquisamos o site da Sociedade Brasileira de Nefrologia (SBN) para obtenção dos dados do Censo Brasileiro de Diálise. Para o cálculo da prevalência, considerou-se a estimativa de pacientes em diálise informada no censo de cada ano como numerador e a estimativa da população do País para aquele ano de acordo com o IBGE ${ }^{21}$.

\section{Seleção dos estudos}

Selecionaram-se as referências relevantes pelos títulos e resumos por dois revisores independentes (AWM e APP) e as discordâncias resolvidas por consenso. Se o resumo estava indisponível, era acessado o texto completo para determinar elegibilidade, a partir dele processo pareado. Utilizou-se o software online Covidence nesta etapa.

\section{Análise e extração de dados}

Um pesquisador fez extração dos dados (AWM) e outros dois a revisão destes (APP, TFG). Coletaram-se as seguintes informações: autor, ano de publicação, país de origem, delineamento, características dos participantes, número de pacientes, idade, prevalência de doença renal crônica, critério diagnóstico. 


\section{Avaliação da qualidade}

Dois pesquisadores avaliaram a qualidade dos artigos selecionados em conjunto (AWM, TFG). Adaptou-se uma ferramenta para avaliação da qualidade metodológica ${ }^{22}$ composta por seis itens (Tabela 1): amostragem apropriada (aleatória, probabilística ou universo); tamanho de amostra adequado (previamente calculado); critérios adequados para avaliação do desfecho (confirmação de alteração estrutural pela proteinúria e/ou funcional pela creatininemia por meio de dosagens com intervalo de pelo menos 3 meses ou pacientes em tratamento dialítico); desfechos mensurados com imparcialidade (pacientes com e sem nefropatia avaliados igualmente); taxa de resposta adequada (> 70\%); participantes similares à pergunta (população adulta $\geq 18$ anos). Cada publicação recebeu pontuação entre 0 e 6 proporcional à sua qualidade metodológica.

\section{Análise dos dados}

O desfecho primário avaliado foi a prevalência de doença renal crônica conforme definido em cada estudo. As diferenças metodológicas dos estudos inviabilizaram a sumarização quantitativa dos resultados por meio de meta-análise, procedendo-se somente a análise descritiva dos resultados individuais.

\section{RESULTADOS}

Localizaram-se 949 referências nas bases bibliográficas, além dos microdados de cinco estudos. Destes, selecionamos 34 para leitura em texto completo, sendo excluídos 18 pelos motivos constantes na Figura 1. Finalmente incluímos 16 estudos $^{23-38}$. Apenas a publicação principal de cada pesquisa é citada para fins de clareza do texto.

\section{Características estudos dos incluídos}

As pesquisas ocorreram entre 1997 e 2015, com predomínio nas regiões Sul e Sudeste (Tabela 2). Neste período, incluíram-se seis estudos de base populacional: três referentes à Pesquisas Nacional por Amostra de Domicílio (PNAD) ${ }^{24,29,32}$; um referente à Pesquisa Nacional de Saúde (PNS) ${ }^{38}$; uma coorte de idosos e adultos realizada em Bambuí23; e um inquérito domiciliar em Salvador ${ }^{26}$. Seis pesquisas incluíram usuários de serviços de saúde: uma avaliou dados de Autorização de Procedimento de Alta Complexidade (APAC) de pacientes em terapia renal substitutiva no Sistema Único de Saúde (SUS) de todo País ${ }^{27}$; outra analisou pacientes internados em um hospital na cidade de São Paulo ${ }^{35}$; outras três avaliaram usuários da Estratégia de Saúde Família de Tubarão ${ }^{34,36}$ e de Goiânia ${ }^{37}$.

Dados do Censo Brasileiro de Diálise, realizado anualmente pela SBN foram incluídos, analisando-se os dados dos pacientes em terapia dialítica no País de 1999 até $2015^{25}$. O censo da SBN provém de dados voluntariamente informados pelas clínicas públicas e privadas do Brasil. A taxa de adesão é variável, tendo sido observada maior resposta em 2007 (87,92\%) e menor em $2012(39,17 \%)^{25}$. A fim de minimizar o efeito da taxa de resposta, a SBN calcula estimativas levando-se em conta os números esperados nos centros que não responderam a cada inquérito, sendo atribuído para o centro um número médio de pacientes esperado na região.

Resultados de campanha da prevenção de insuficiência renal realizados em Curitiba ${ }^{28}$ e no estado de São Paulo foram relatados ${ }^{31}$. Também foi incluída uma coorte de adultos saudáveis, o Estudo Longitudinal de Saúde do Adulto (ELSA) ${ }^{33}$, composto por servidores ativos e aposentados de instituições de ensino e pesquisa de seis capitais brasileiras (São Paulo, Salvador, Belo

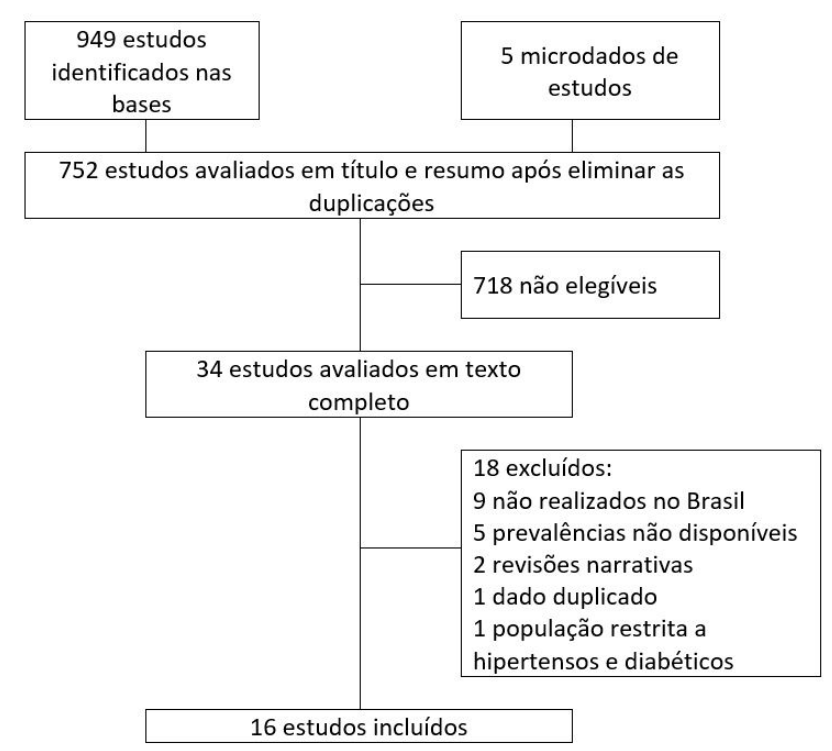

Figura 1. Processo de seleção dos estudos elegíveis

Tabela 1. Critérios utilizados para avaliação crítica dos estudos que estimaram a prevalência de doença renal crônica (adaptado de Loney et al., 1998)22

\section{Descrição}

Amostragem apropriada

Tamanho de amostra adequado

Critérios adequados para avaliação do desfecho

Desfechos mensurados com imparcialidade

Taxa de resposta adequada

Participantes similares à pergunta

\section{Critério considerado como adequado}

Aleatória, probabilística ou universo

Tamanho de amostra previamente calculado

Confirmação de alteração estrutural (proteinúria) e/ou funcional (creatininemia), por meio de dosagens com intervalo de pelo menos 3 meses;

Pacientes em tratamento dialítico

Pacientes com e sem nefropatia avaliados igualmente

Recusas ou perdas $<30 \%$ (taxa de resposta $>70 \%$ )

População adulta ( $\geq 18$ anos) 
Tabela 2. Características epidemiológicas dos estudos incluídos

\begin{tabular}{|c|c|c|c|c|c|c|}
\hline Estudo & Ano & Local & Fonte & $\begin{array}{l}\text { População } \\
\text { (n) }\end{array}$ & $\begin{array}{c}\text { Sexo } \\
\text { feminino (\%) }\end{array}$ & $\begin{array}{l}\text { Faixa etária } \\
\text { (anos) }\end{array}$ \\
\hline Passos et al. $(2003)^{23}$ & 1997 & Bambuí, MG & $\begin{array}{l}\text { Coorte populacional } \\
\text { (Projeto Bambuí) }\end{array}$ & 2.312 & $60,77^{\mathrm{a}}$ & Adultos $(\geq 18)$ \\
\hline PNAD $(1998)^{24}$ & 1998 & Brasil & Inquérito domiciliar & 114.045 & 64,97 & Adultos ( $\geq 18)$ \\
\hline Censo SBN $(2015)^{25}$ & 1999-2015 & Brasil & Censo Brasileiro de Diálise & 1.278 .314 & $42,00^{\mathrm{b}}$ & $\begin{array}{l}\text { Crianças e } \\
\text { adultos }(\geq 0)\end{array}$ \\
\hline Lessa $(2004)^{26}$ & 2000 & Salvador, BA & Inquérito domiciliar & 1.439 & 57,68 & Adultos $(\geq 20)$ \\
\hline Moura et al. $(2014)^{27}$ & 2000-2012 & Brasil & $\begin{array}{l}\text { Sistema Único de Saúde } \\
\text { (APAC) }\end{array}$ & 280.667 & 42,79 & $\begin{array}{l}\text { Crianças e } \\
\text { adultos }(\geq 0)\end{array}$ \\
\hline Nascimento e Riella (2009) ${ }^{28}$ & $2002-2005$ & Curitiba, PR & Campanha em feira de saúde & 8.883 & 55,99 & Adultos $(\geq 17)$ \\
\hline PNAD $(2003)^{29}$ & 2003 & Brasil & Inquérito domiciliar & 134.397 & 65,53 & Adultos ( $\geq 18)$ \\
\hline Bastos et al. $(2009)^{30}$ & 2004-2005 & Juiz de Fora, MG & Registro laboratorial & 24.248 & 59,62 & Adultos (>18) \\
\hline Lima et al. $(2012)^{31}$ & $2005-2010$ & São Paulo & Campanha em feira de saúde & 37.771 & 55,74 & $\begin{array}{l}\text { Crianças e } \\
\text { adultos }(\geq 0)\end{array}$ \\
\hline PNAD $(2008)^{32}$ & 2008 & Brasil & Inquérito domiciliar & 169.194 & 61,25 & Adultos $(\geq 18)$ \\
\hline Barreto et al. $(2016)^{33}$ & $2008-2010$ & Brasil $^{c}$ & Coorte institucional (ELSA) & 14.636 & 54,10 & Adultos ( $\geq 35)$ \\
\hline Dutra et al. $(2014)^{34}$ & $2010-2011$ & Tubarão, SC & Estratégia saúde da família & 822 & 61,56 & $\operatorname{Idosos}(\geq 60)$ \\
\hline Pinho et al. $(2015)^{35}$ & $2010-2013$ & São Paulo, SP & Prontuários hospitalares & 386 & 49,48 & Adultos $(\geq 18)$ \\
\hline Schaefer et al. $(2015)^{36}$ & 2011-2012 & Tubarão, SC & Estratégia saúde da família & 371 & 63,61 & Adultos (18-59) \\
\hline Pereira et al. $(2016)^{37}$ & 2011-2013 & Goiânia, GO & Estratégia saúde da família & 220 & $67,71^{\mathrm{d}}$ & Adultos $(\geq 20)$ \\
\hline PNS $(2013)^{38}$ & 2013 & Brasil & Inquérito domiciliar & 45.236 & 61,30 & Adultos $(\geq 18)$ \\
\hline
\end{tabular}

Notas: PNAD, Pesquisa Nacional por Amostra de Domicílio; APAC, Autorização de Procedimento de Alta Complexidade; SBN, Sociedade Brasileira de Nefrologia;

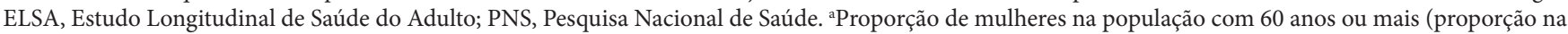
população total indisponível); ${ }^{b}$ Proporção de mulheres no ano de 2015 (proporção para todo o período indisponível); 'Salvador, Belo Horizonte, Rio de Janeiro,

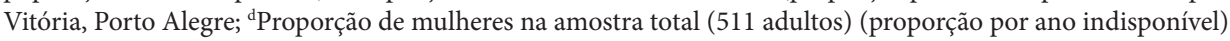

Horizonte, Rio de Janeiro, Vitória e Porto Alegre) e um registro de resultados de um laboratório de análises clínicas privado ${ }^{30}$.

\section{Avaliação da qualidade}

A maior parte dos estudos empregou amostragem por conveniência, não representativa da população em geral ${ }^{25,27,28,30,31,33-37}$ (Tabela 3). Também foi comum como a mensuração inadequada dos desfechos, utilizando o autorrelato dos pacientes ${ }^{24,29,32,38}$ ou por dosagens laboratoriais em episódio único ${ }^{23,26,28,31,33,34,36,37}$. Não houve cálculo prévio do tamanho da amostra em cinco pesquisas ${ }^{25,27,28,30,31}$. Nenhum estudo atendeu a todos os critérios de qualidade, em geral apresentando vieses na seleção da população ou na aferição do desfecho. As pesquisas que estimaram os pacientes em terapia dialítica incluíram, em menor proporção, a população pediátrica, tendo seu escore rebaixado por este motivo $^{25,27}$. Optou-se por manter estas pesquisas na análise, apesar do risco de evidência indireta por trazerem estimativas da proporção de pacientes em diálise no País.

\section{Prevalência de doença renal crônica}

\section{Estudos populacionais}

As pesquisas realizadas pelo IBGE mensuraram de forma autorreferida, com as perguntas "O Sr./Sra. tem doença renal?" (1998) e "Algum médico ou profissional de saúde disse que o Sr./Sra. tem doença renal?” (a partir de 2003), que poderiam ser respondidas com "sim" ou "não" (Tabela 4). A prevalência autorreferida no intervalo de 15 anos dos inquéritos apresentou tendência decrescente, com relatada prevalência de $4,57 \%$ em $1998^{24}, 2,87 \%$ em $2003^{29}, 1,90 \%$ em $2008^{32}$ e $1,43 \%$ em $2013^{38}$.

Os outros de base populacional utilizaram a hipercreatininemia como critério diagnóstico. A coorte de idosos de Bambuí $(\mathrm{N}=2.312)$ realizou uma etapa transversal com a inclusão de adultos maiores de 18 anos: 3,46\% apresentaram creatinina sérica elevada, sendo maior nos idosos do sexo masculino ${ }^{23}$. O inquérito populacional realizado em 1.439 adultos de Salvador no ano 2000 encontrou 3,13\% de hipercreatininemia, com maior prevalência no sexo masculino ${ }^{26}$.

\section{Estudos institucionais e campanhas}

Nos 14.636 funcionários públicos incluídos no ELSA, encontrou prevalência de $8,94 \%$ pela análise da taxa de filtração glomerular e/ou relação albuminúria-creatinina urinária ${ }^{33}$. As aferições ocorreram de 2008 a 2010 e incluíram pessoas de 34 a 74 anos, com leve predominância de mulheres (54,10\%).

Campanhas realizadas em 37.771 adultos (55,74\% mulheres) no estado de São Paulo entre 2005 e 2010 encontraram prevalência de 7,26\% de proteinúria entre os participantes submetidos ao teste da fita reagente em amostra de urina ${ }^{31}$. Em Curitiba, no período de 2002 a 2005, esta mesma abordagem encontraram $6,00 \%$ de proteinúria em 8.883 adultos $\geq 17$ anos, dos quais $55,99 \%$ eram mulheres ${ }^{28}$. 
Tabela 3. Avaliação da qualidade dos estudos incluídos

\begin{tabular}{|c|c|c|c|c|c|c|c|}
\hline Estudo & $\begin{array}{c}\text { Amostragem } \\
\text { apropriada }\end{array}$ & $\begin{array}{c}\text { Tamanho da } \\
\text { amostra }\end{array}$ & $\begin{array}{c}\text { Avaliação do } \\
\text { desfecho }\end{array}$ & $\begin{array}{l}\text { Avaliação } \\
\text { imparcial }\end{array}$ & $\begin{array}{l}\text { Taxa de } \\
\text { resposta }\end{array}$ & $\begin{array}{c}\text { Participantes } \\
\text { semelhantes }\end{array}$ & Escore \\
\hline Passos et al. $(2003)^{23}$ & 1 & 1 & $0^{\mathrm{a}}$ & 1 & 1 & $0^{\mathrm{b}}$ & 4 \\
\hline PNAD $(1998)^{24}$ & 1 & 1 & $0^{\mathrm{a}}$ & 1 & 1 & 1 & 5 \\
\hline Censo SBN $(2015)^{25}$ & $0^{c}$ & $0^{\mathrm{d}}$ & 1 & $0^{\mathrm{e}}$ & $0^{\mathrm{f}}$ & $0^{\mathrm{g}}$ & 1 \\
\hline Lessa $(2004)^{26}$ & 1 & 1 & $0^{\mathrm{a}}$ & 1 & 1 & 1 & 5 \\
\hline Moura et al. $(2014)^{27}$ & $0^{c}$ & $0^{\mathrm{d}}$ & 1 & $0^{\mathrm{e}}$ & 1 & $0^{\mathrm{f}}$ & 2 \\
\hline Nascimento e Riella (2009) ${ }^{28}$ & $0^{c}$ & $0^{\mathrm{d}}$ & $0^{\mathrm{a}}$ & 1 & 1 & 1 & 3 \\
\hline PNAD $(2003)^{29}$ & 1 & 1 & $0^{\mathrm{a}}$ & 1 & 1 & 1 & 5 \\
\hline Bastos et al. (2009) ${ }^{30}$ & $0^{c}$ & $0^{\mathrm{d}}$ & 1 & $0^{\mathrm{h}}$ & 1 & $0^{\mathrm{i}}$ & 2 \\
\hline Lima et al. $(2012)^{31}$ & $0^{c}$ & $0^{\mathrm{d}}$ & $0^{\mathrm{a}}$ & 1 & 1 & 1 & 3 \\
\hline PNAD $(2008)^{32}$ & 1 & 1 & $0^{\mathrm{a}}$ & 1 & 1 & 1 & 5 \\
\hline Barreto et al. $(2016)^{33}$ & $0^{c}$ & 1 & $0^{\mathrm{a}}$ & 1 & 1 & 1 & 4 \\
\hline Dutra et al. $(2014)^{34}$ & $0^{c}$ & 1 & $0^{\mathrm{a}}$ & 1 & $0^{j}$ & $0^{\mathrm{b}}$ & 2 \\
\hline Pinho et al. $(2015)^{35}$ & $0^{c}$ & 1 & 1 & 1 & 1 & 1 & 5 \\
\hline Schaefer et al. $(2015)^{36}$ & $0^{c}$ & 1 & $0^{\mathrm{a}}$ & 1 & $0^{j}$ & 1 & 3 \\
\hline Pereira et al. $(2016)^{37}$ & $0^{c}$ & 1 & 1 & 1 & 1 & $0^{\mathrm{b}}$ & 4 \\
\hline PNS $(2013)^{38}$ & 1 & 1 & $0^{\mathrm{a}}$ & 1 & 1 & 1 & 5 \\
\hline Total de itens atendidos & 6 & 11 & 5 & 13 & 13 & 10 & - \\
\hline
\end{tabular}

Notas: PNAD, Pesquisa Nacional por Amostra de Domicílio; SBN, Sociedade Brasileira de Nefrologia; PNS, Pesquisa Nacional de Saúde. ${ }^{2}$ Avaliação do desfecho

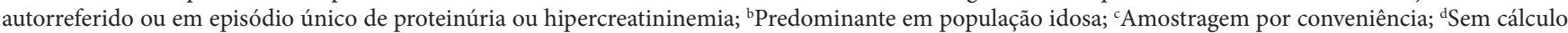
do tamanho da amostra; ${ }^{\mathrm{e}} \mathrm{O}$ denominador igual à população anual do País, não sendo avaliado o desfecho em toda a população brasileira; ${ }^{\mathrm{f}} \mathrm{Dados}$ informados voluntariamente pelas clínicas de diálise em questionário on-line. Em 2014, a taxa de resposta na Região Norte foi 5\% e na Sudeste, $47 \%{ }^{39}$; g Incluiu pessoas de 0 a 19 anos (menos de 6\% da amostra no Censo $\mathrm{SBN}^{25}$ e 3,4\% no estudo Moura et al. ${ }^{27}$ ); ${ }^{\text {h}} \mathrm{A}$ segunda dosagem da creatinina buscada somente naqueles com

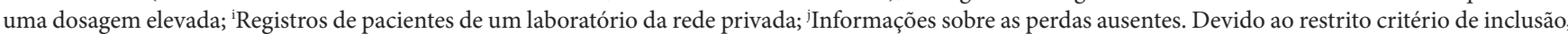
assumiu-se que foram importantes (pacientes tinham que comparecer em jejum aos sábados de manhã após 12 horas de jejum e abstinência de álcool por 72 horas)

\section{Estudos com usuários de serviços de saúde}

Dados retrospectivos de um hospital universitário de São Paulo com 386 pacientes $\geq 18$ anos, 49,48\% sexo feminino, encontrou prevalência de $27,20 \%$. O diagnóstico baseou-se no registro médico em prontuários de pacientes que tinham duas dosagens de creatinina sérica durante a internação em uma enfermaria no período de 2010 a $2013^{35}$.

Na Estratégia de Saúde Família de Goiânia com 220 adultos $\geq 20$ anos, $67,71 \%$ sexo feminino, observou-se $10,64 \%$ de pacientes com taxa de filtração glomerular $\leq 60 \mathrm{ml} / \mathrm{min} / 1,73 \mathrm{~m}^{237}$. O Estudo Saúde dos Idosos de Tubarão incluiu 822 adultos com 60 anos ou mais, $61,56 \%$ do sexo feminino, $92,2 \%$ brancos, e obteve $13,63 \%$ com taxa de filtração glomerular alterada ${ }^{34}$. A mesma investigação com 371 adultos de 18 a 59 anos, 63,61\% mulheres, $86,3 \%$ brancos, encontrou prevalência de $1,35 \%{ }^{36}$. Nestas duas pesquisas, os pacientes cadastrados na Estratégia de Saúde Família de Tubarão compareceram aos sábados, respeitando jejum de 12 horas e abstinência alcoólica de 72 horas, no Centro de Pesquisas Clínicas do Hospital Nossa Senhora da Conceição, Tubarão, para realizar as dosagens.

Análise de 24.248 clientes de um laboratório de análises clínicas de Juiz de Fora encontrou prevalência de 9,62\% avaliada pela taxa de filtração glomerular ${ }^{30}$. A maior parte era do sexo feminino (59,62\%), com média de idade 48-74 anos.
A prevalência de diálise reembolsada pelo SUS entre os anos 2000 e 2012 variou de $0,03 \%$ em 2000 (336,3 pacientes por milhão da população [pmp]) a 0,05\% em 2012 (538,3 pmp), por meio de pareamento de dados das APAC ${ }^{27}$. Para cálculo dessa prevalência, os pesquisadores empregaram como denominador a estimativa da população anual do Brasil. Realizam mais diálise pessoas do sexo masculino $(57,2 \%)$, brancas $(45,2 \%)$ e com idade 45-64 anos (43,4\%), com predomínio da hemodiálise como modalidade terapêutica $(90,1 \%)$ e diálise peritoneal em menor proporção (9,9\%). Negros $(9,7 \%)$ e pardos $(30,8 \%)$ tiveram menor representatividade dentre os pacientes em diálise. Entre as principais causas da doença destaca-se hipertensão arterial sistêmica (34\%), diabetes melito 29\% e glomerulopatias (13\%). Crianças de 0 a 19 anos representaram 1,3\% da população em terapia dialítica no $\mathrm{SUS}^{27}$.

No intervalo de 2005 a 2015, a prevalência estimada pelo censo da SBN dos pacientes em diálise praticamente dobrou, passando de 0,03\% (294,9 pmp) para 0,05\% (544 pmp $)^{25}$. Neste período também observou-se redução na prevalência de hepatite B, que passou de $1,80 \%$ em 2005 para 0,90\% em 2015, e de hepatite $\mathrm{C}$, que reduziu de $11,00 \%$ para $3,80 \%$. A prevalência de pacientes portadores do vírus da imunodeficiência humana aumentou de $0,50 \%$ para $0,80 \%$. Hemodiálise correspondeu à terapia dialítica mais empregada em todos os anos e em 2015 atingiu $92,80 \%$, a maior parte com reembolso pelo SUS, $84,00 \%$. Os mais acometidos são os adultos entre 20 e 65 anos de idade, 
Tabela 4. Prevalência de doença renal crônica e critérios diagnósticos adotados nos estudos

\begin{tabular}{|c|c|c|c|}
\hline Estudo & Critério diagnóstico & Definição de doença renal crônica & Prevalência (\%) \\
\hline \multicolumn{4}{|l|}{ Populacionais } \\
\hline PNAD $(1998)^{24}$ & Autorreferido & O Sr./Sra. tem doença renal? & 4,57 \\
\hline PNAD $(2003)^{29}$ & Autorreferido & $\begin{array}{l}\text { Algum médico ou profissional de saúde disse que o Sr./Sra. } \\
\text { tem doença renal? }\end{array}$ & 2,87 \\
\hline PNAD $(2008)^{32}$ & Autorreferido & $\begin{array}{l}\text { Algum médico ou profissional de saúde disse que o Sr./Sra. } \\
\text { tem doença renal? }\end{array}$ & 1,90 \\
\hline PNS $(2013)^{38}$ & Autorreferido & $\begin{array}{l}\text { Algum médico ou profissional de saúde disse que o Sr./Sra. } \\
\text { tem doença renal? }\end{array}$ & 1,43 \\
\hline Passos et al. $(2003)^{23}$ & $\begin{array}{l}\text { Creatinina sérica } \\
\text { (única dosagem) }\end{array}$ & $\begin{array}{l}\text { Creatinina sérica } \geq 1,3 \mathrm{mg} / \mathrm{dl} \text { para homens e } \geq 1,1 \mathrm{mg} / \mathrm{dl} \\
\text { para mulheres }\end{array}$ & 3,46 \\
\hline Lessa $(2004)^{26}$ & $\begin{array}{l}\text { Creatinina sérica } \\
\text { (única dosagem) }\end{array}$ & Creatinina sérica $\geq 1,3 \mathrm{mg} / \mathrm{dl}$ & 3,13 \\
\hline \multicolumn{4}{|l|}{ Institucional } \\
\hline Barreto et al. $(2016)^{33}$ & $\begin{array}{l}\text { Creatinina sérica e/ou } \\
\text { proteinúria (única dosagem) }\end{array}$ & $\begin{array}{l}\text { Taxa de filtração glomerular }<60 \mathrm{ml} / \mathrm{min} / 1.73 \mathrm{~m}^{2} \text { e/ou } \\
\text { relação albumina-creatinina } \geq 30 \mathrm{mg} / \mathrm{g}\end{array}$ & 8,94 \\
\hline \multicolumn{4}{|l|}{ Campanhas } \\
\hline Nascimento e Riella (2009) ${ }^{28}$ & Proteinúria & Fita reativa positiva $(1 \mathrm{a} 4+)$ & 6,00 \\
\hline Lima et al. $(2012)^{31}$ & Proteinúria & Fita reativa positiva $(1 \mathrm{a} 4+)$ & 7,26 \\
\hline \multicolumn{4}{|l|}{ Serviços de saúde } \\
\hline \multicolumn{4}{|l|}{ Atenção primária } \\
\hline Dutra et al. $(2014)^{34}$ & $\begin{array}{l}\text { Creatinina sérica } \\
\text { (única dosagem) }\end{array}$ & Taxa de filtração glomerular $<60 \mathrm{ml} / \mathrm{min} / 1,73 \mathrm{~m}^{2}$ & 13,63 \\
\hline Schaefer et al. $(2015)^{36}$ & $\begin{array}{l}\text { Creatinina sérica } \\
\text { (única dosagem) }\end{array}$ & Taxa de filtração glomerular $<60 \mathrm{ml} / \mathrm{min} / 1,73 \mathrm{~m}^{2}$ & 1,35 \\
\hline Pereira et al. $(2016)^{37}$ & $\begin{array}{l}\text { Creatinina sérica e/ou } \\
\text { proteinúria (única dosagem) }\end{array}$ & Taxa de filtração glomerular $<60 \mathrm{ml} / \mathrm{min} / 1,73 \mathrm{~m}^{2}$ & 10,64 \\
\hline \multicolumn{4}{|l|}{ Laboratório } \\
\hline Bastos et al. $(2009)^{30}$ & $\begin{array}{l}\text { Creatinina sérica } \\
\text { (duas dosagens) }\end{array}$ & Taxa de filtração glomerular $<60 \mathrm{ml} / \mathrm{min} / 1,73 \mathrm{~m}^{2}$ & 9,62 \\
\hline \multicolumn{4}{|l|}{ Hospital } \\
\hline Pinho et al. $(2015)^{35}$ & $\begin{array}{l}\text { Creatinina sérica } \\
\text { (duas dosagens) }\end{array}$ & $\begin{array}{l}\text { Registro médico no prontuário de pacientes com } 2 \\
\text { dosagens de creatinina sérica }\end{array}$ & 27,20 \\
\hline \multicolumn{4}{|l|}{ Clínicas de diálise } \\
\hline Censo SBN $(2015)^{25}$ & Diagnóstico clínico & Pacientes em diálise em clínicas públicas e privadas & $0,03-0,06^{\mathrm{a}}$ \\
\hline Moura et al. $(2014)^{27}$ & Diagnóstico clínico & Pacientes em diálise no SUS & $0,03-0,05^{\mathrm{b}}$ \\
\hline
\end{tabular}

Notas: PNAD, Pesquisa Nacional por Amostra de Domicílio; SBN, Sociedade Brasileira de Nefrologia; SUS, Sistema Único de Saúde; PNS, Pesquisa Nacional de Saúde. ${ }^{a}$ Prevalência, \% (paciente por milhão da população): $1999=0,03(254,3) ; 2000=0,03(274,2) ; 2001=0,03(283,1) ; 2002=0,03(312,2) ; 2003=0,03(334,4)$; $2004=0,03(326,4) ; 2005=0,03(294,9) ; 2006=0,04(379,5) ; 2007=0,04(388,8) ; 2008=0,05(459,1) ; 2009=0,05(405,2) ; 2010=0,05(482,8) ; 2011=0,05(474,7)$; $2012=0,05(503,2) ; 2013=0,05(499,4) ; 2014=0,06(552,4) ; 2015=0,05(544,0)$; ${ }^{b}$ Prevalência, \% (paciente por milhão da população): $2000=0,03(336,3)$; $2001=0,04(366,2) ; 2002=0,04(376,0) ; 2003=0,04(401,8) ; 2004=0,04(416,7) ; 2005=0,04(435,7) ; 2006=0,05(425,0) ; 2007=0,05(466,3) ; 2008=0,05(459,1)$; $2009=0,05(484,7) ; 2010=0,05(502,8) ; 2011=0,05(521,4) ; 2012=0,05(538,3)$

que representaram 64,90\% no ano de 2015; sexo masculino foi o mais prevalente para todos os anos, e em 2015 representou $58 \%$ dos pacientes em diálise ${ }^{25}$.

\section{DISCUSSÃO}

A prevalência de doença renal crônica na população brasileira ainda é incerta. Estimativas populacionais mais recentes revelam cerca de $1,5 \%$ de doença renal autorreferida. A prevalência de hipercreatininemia na população ficou em torno de 3\%. A partir destes dados, de 3 a 6 milhões de adultos teriam a doença. Em relação a acesso ao tratamento, 0,05\% da população brasileira realiza dialítica, o que representaria em torno de 100 mil pacientes.
As prevalências encontradas são provenientes de estudos transversais e de coorte aplicados em amostras de base populacional ou amostras por conveniência (registros laboratoriais, campanhas de sensibilização ou provenientes de instituições). Pesquisas que usaram os melhores critérios diagnósticos partiram de amostras selecionadas, sem representatividade da população: estudos de melhor validade interna para o diagnóstico tiveram baixa capacidade de generalização. Como resultado dessa baixa validade externa, tiveram as maiores prevalências ${ }^{23,30,34,36,37}$.

Inquéritos com amostragem representativa mensuraram a doença pelo autorrelato ou pela hipercreatininemia isolada ${ }^{23,24,26,29,32,38}$. Para confirmar a doença renal crônica, seriam necessárias pelo menos duas dosagens seriadas da creatinina ao longo de três 
meses ou comparar com outras dosagens anteriores ${ }^{39}$, tempo necessário para definição e diferenciação entre disfunção aguda e crônica ${ }^{2}$.

Além das questões referentes à seleção da amostra e mensuração do desfecho, as pesquisas incluíam pacientes de diferentes faixas etárias, sem informar consistentemente a prevalência em cada faixa. É conhecido que a prevalência de nefropatias aumenta com a idade ${ }^{1,40}$. Em menor escala, mas de semelhante relevância, as diferenças naturais entre os sujeitos incluídos em cada análise, considerou-se a diversidade socioeconômica, demográfica e cultural da população brasileira, podem também influenciar nos resultados encontrados ${ }^{41}$. A heterogeneidade entre pesquisas inviabilizou a elaboração de meta-análise, cujo resultado seria de baixa aplicabilidade, dadas essas limitações.

Estimar a real prevalência na população ainda é um desafio devido ao critério diagnóstico. A dosagem da creatinina sérica e a pesquisa de albuminúria são os principais marcadores empregados. Com estes marcadores a prevalência chegou a 13,1\% em adultos no último National Health and Nutrition Examination Survey (NHANES, 1999-2004 $\mathrm{n}=13.233)^{42}$. Em uma coorte chinesa com 47.204 adultos de 13 províncias, entre 2009 e 2010, obteve-se a prevalência de $10,8 \%{ }^{4}$. Nos demais países emergentes, estima-se que a prevalência seja igualmente elevada $^{41,43}$.

Os doentes renais apresentam alto risco de eventos cardiovasculares fatais e mortalidade mesmo antes de atingir os estágios avançados ${ }^{44}$. Esse viés de sobrevivência influencia nos achados observacionais, que pode representar inadequadamente a população doente por apenas incluir aqueles que acessaram $o$ tratamento em tempo hábil. A progressão de nefropatias sem tratamento adequado aumenta o risco de desfechos negativos mortalidade $e^{40,45,46}$.

A presença de sintomas urêmicos e necessidade de diálise apontam para o diagnóstico tardio ${ }^{39}$. Todavia, o caráter insidioso dos estágios iniciais dificulta o diagnóstico precoce da doença renal crônica ${ }^{1}$. Tal fato pode explicar a baixa prevalência autorreferida nos estudos incluídos ${ }^{24,29,32,38}$. A prevalência informada reflete os casos diagnosticados e que tiveram acesso a serviços de saúde ${ }^{43}$.

O número de pacientes em terapia dialítica na América Latina tem aumentado nos últimos anos, o que pode estar associado às dificuldades de acesso ao tratamento $0^{2,13,47,48}$. Dados sobre estágios iniciais permanecem indisponíveis ou pouco explorados nestes países $^{48}$, possivelmente devido à falta de diagnóstico precoce. Observa-se uma avançada industrialização nessas regiões, acompanhadas do aumento na prevalência de hipertensão, diabetes e doença cardiovascular, sobretudo em setores de baixo nível socioeconômico ${ }^{48-50}$. Além disso, deficiências nos sistemas de saúde e na promoção de medidas preventivas sabidamente favorecem evolução para disfunção crônica ${ }^{14,51,52}$. As disparidades sociais e de saúde figuram importantes problemas no Brasil, a despeito das melhorias no acesso ao sistema de saúde e qualidade dos serviços observados nas últimas décadas ${ }^{41}$. As discrepâncias observadas entre portadores de disfunção crônica estão relacionadas principalmente ao nível de escolaridade, gênero, região geográfica e raça ${ }^{53}$.

A proporção de negros, pardos e indígenas entre os brasileiros em terapia renal substitutiva é baixa, indicando menor sobrevivência ou dificuldades de acesso a serviços de saúde ${ }^{53,54}$. A insuficiência renal está associada aos menores índices de escolaridade, ao passo que aqueles com melhor nível educacional são os que mais têm acesso ao tratamento dialítico e/ou transplante ${ }^{55}$. O baixo nível socioeconômico está associado à microalbuminúrica, macroalbuminúria, redução da taxa de filtração glomerular e perda progressiva de função dos néfron $s^{56}$. Tais aspectos evidenciam a iniquidade da assistência no País.

Os inquéritos populacionais realizados no Brasil tiveram sua abrangência geográfica gradativamente ampliada. Os resultados das PNAD de 1998 a 2003 representam as áreas urbanas das Unidades da Federação e grandes regiões metropolitanas, exceto as zonas rurais de seis estados da Região Norte ${ }^{24,29}$. Somente a partir de 2004 a PNAD alcançou a cobertura completa do território nacional ${ }^{57}$. Além da maior cobertura, a abordagem e questionamento a respeito da condição de portador de insuficiência renal crônica sofreu modificação ao longo dos anos. A inclusão tardia desses extratos pode explicar a variação na prevalência de autorrelato observada ao longo dos anos. A maior disponibilidade de serviços de saúde, sobretudo nas Regiões Sul e Sudeste, potencialmente aumentam o conhecimento da população sobre sua condição clínica, facilitam o acesso ao diagnóstico e ao tratamento de estágios avançados ${ }^{49,58}$.

No Brasil, os pacientes renais recebem tratamento dialítico majoritariamente por meio do SUS ${ }^{27}$. A diálise é considerada um tratamento de alto custo e no Brasil o SUS é responsável pelo reembolso de quase $90 \%$ dessa terapia ${ }^{27}$. Apesar disso, é desconhecida a frequência de pacientes que morrem antes terem acesso à diálise. Tampouco se sabe a frequência de pacientes submetidos ao transplante renal sem diálise prévia, chamado transplante preemptivo ${ }^{59}$.

A crise econômica que o Brasil enfrenta desestimula a abertura de novas clínicas e favorece o fechamento das unidades existentes ${ }^{60}$. Atualmente observa-se redução no número de vagas para o tratamento do doente e aumento da lista de espera por tratamento nos centros de diálise $e^{61-63}$.

$\mathrm{Na}$ atenção básica de saúde, o adequado tratamento e controle do diabetes e da hipertensão podem reduzir os fatores de risco para instalação e progressão para cronicidade ${ }^{64}$. Nos estudos incluídos que avaliaram usuários deste nível de atenção, foram observadas prevalências elevadas ${ }^{34,36,37}$. O rastreamento neste grupo pela albuminúria, por exemplo, seria uma medida 
importante para prevenir a progressão ${ }^{52}$. No Brasil, os hospitais e serviços de emergências continuam como porta de entrada para o tratamento dialítico ${ }^{52}$, invertendo a lógica da atenção primária em saúde.

A presente revisão seguiu de forma rigorosa as recomendações para elaboração de revisões sistemáticas: busca estruturada da literatura disponível, sem restrição de idioma ou ano de publicação, seleção e extração pareada de dados, e avaliação da qualidade metodológica ${ }^{65,66}$. Tais recomendações são essenciais para dirimir os possíveis vieses e aumentar a transparência nos resultados apresentados, para possibilitar sua reprodutibilidade.

\section{REFERÊNCIAS}

1. Glassock RJ, Winearls C. The global burden of chronic kidney disease: how valid are the estimates? Nephron Clin Pract. 2008;110(1):c39-47. PMid:18689986. http://dx.doi.org/10.1159/000151244.

2. Francis ER, Kuo CC, Bernabe-Ortiz A, Nessel L, Gilman RH, Checkley $\mathrm{W}$, et al. Burden of chronic kidney disease in resource-limited settings from Peru: a population-based study. BMC Nephrol. 2015;16:114. PMid:26205002. http://dx.doi.org/10.1186/s12882-015-0104-7.

3. Stanifer JW, Muiru A, Jafar TH, Patel UD. Chronic kidney disease in low and middle-income countries. Nephrol Dial Transplant. 2016;31(6):868-74. PMid:27217391. http://dx.doi.org/10.1093/ndt/gfv466.

4. Zhang L, Wang F, Wang L, Wang W, Liu B, Liu J, et al. Prevalence of chronic kidney disease in China: a cross-sectional survey. Lancet. 2012;379(9818):81522. PMid:22386035. http://dx.doi.org/10.1016/S0140-6736(12)60033-6.

5. Bortolotto LA. Hipertensão arterial e insuficiência renal crônica. Rev Bras Hipertens. 2008;15(3):152-5.

6. National Kidney Foundation. KDOQI clinical practice guideline for diabetes and CKD: 2012 update. Am J Kidney Dis. 2012;60(5):850-86. PMid:23067652. http://dx.doi.org/10.1053/j.ajkd.2012.07.005.

7. Becker G, Wheeler D, Zeeuw D. KDIGO Clinical practice guideline for the management of blood pressure in chronic kidney disease. Kidney Int. 2012;2:S337-414.

8. Franceschini N, Gouskova NA, Reiner AP, Bostom A, Howard BV, Pettinger $\mathrm{M}$, et al. Adiposity patterns and the risk for ESRD in postmenopausal women. Clin J Am Soc Nephrol. 2015;10(2):241-50. PMid:25452225. http:// dx.doi.org/10.2215/CJN.02860314.

9. Carrero JJ. Gender differences in chronic kidney disease: underpinnings and therapeutic implications. Kidney Blood Press Res. 2010;33(5):383-92. PMid:20948227. http://dx.doi.org/10.1159/000320389.

10. Abboud H, Henrich WL. Stage IV chronic kidney disease. N Engl J Med. 2010;362(1):56-65. PMid:20054047. http://dx.doi.org/10.1056/ NEJMcp0906797.

11. National Collaborating Centre for Chronic Conditions (UK). Chronic kidney disease: national clinical guideline for early identification and management in adults in primary and secondary care. London: Royal College of Physicians (UK); 2008. PMID: 21413194.
Em conclusão, não há estimativa precisa do número de brasileiros com insuficiência renal crônica entre os brasileiros. Estudos que tiveram melhor representatividade falharam na aferição da doença, e aqueles que aferiram adequadamente partiram de populações pré-selecionadas. De acordo com os inquéritos populacionais, de 3 a 6 milhões de brasileiros seriam renais crônicos. Pouco mais de 100 mil recebem terapia dialítica. Os achados apontam para a necessidade de ampliação do acesso aos serviços de saúde, a fim de diagnosticar precocemente e possibilitar tratamento oportuno. Pesquisas futuras com adequada validade interna e externa são necessárias para estimar a real prevalência de doença renal crônica na população brasileira.
12. Levey AS, Eckardt KU, Tsukamoto Y, Levin A, Coresh J, Rossert J, et al. Definition and classification of chronic kidney disease: a position statement from Kidney Disease: Improving Global Outcomes (KDIGO). Kidney Int. 2005;67(6):2089-100. PMid:15882252. http://dx.doi.org/10.1111/j.15231755.2005.00365.x

13. Ene-Iordache B, Perico N, Bikbov B, Carminati S, Remuzzi A, Perna A, et al. Chronic kidney disease and cardiovascular risk in six regions of the world (ISN-KDDC): a cross-sectional study. Lancet Glob Health. 2016;4(5):e30719. PMid:27102194. http://dx.doi.org/10.1016/S2214-109X(16)00071-1.

14. Bastos MG, Kirsztajn GM. Chronic kidney disease: importance of early diagnosis, immediate referral and structured interdisciplinary approach to improve outcomes in patients not yet on dialysis. J Bras Neurol. 2011;33(1):93-108. PMid:21541469.

15. Gilbertson DT, Liu J, Xue JL, Louis TA, Solid CA, Ebben JP, et al. Projecting the number of patients with end-stage renal disease in the United States to the year 2015. J Am Soc Nephrol. 2005;16(12):3736-41. PMid:16267160. http://dx.doi.org/10.1681/ASN.2005010112.

16. Inker LA, Astor BC, Fox CH, Isakova T, Lash JP, Peralta CA, et al. KDOQI US commentary on the 2012 KDIGO clinical practice guideline for the evaluation and management of CKD. Am J Kidney Dis. 2014;63(5):713-35. PMid:24647050. http://dx.doi.org/10.1053/j.ajkd.2014.01.416.

17. Park JI, Baek H, Jung HH. Prevalence of Chronic Kidney Disease in Korea: the Korean National Health and Nutritional Examination Survey 2011-2013. J Korean Med Sci. 2016;31(6):915-23. PMid:27247501. http:// dx.doi.org/10.3346/jkms.2016.31.6.915.

18. Forni OV, Ogna A, Ponte B, Gabutti L, Binet I, Conen D, et al. Prevalence and determinants of chronic kidney disease in the Swiss population. Swiss Med Wkly. 2016;146:w14313. PMID: 27152492. http://dx.doi.org/10.4414/ smw.2016.14313.

19. Collins AJ, Foley RN, Gilbertson DT, Chen S-C. United States Renal Data System public health surveillance of chronic kidney disease and end-stage renal disease. Kidney Int Suppl. 2015;5(1):2-7. PMid:26097778. http:// dx.doi.org/10.1038/kisup.2015.2.

20. Instituto Brasileiro de Geografia e Estatística [Internet]. Rio de Janeiro: IBGE; 2017 [citado em 2017 Jul 4]. Disponível em: www.ibge.gov.br

21. Eknoyan G, Lameire N, Eckardt K, Kasiske B, Wheeler D, Levin A, et al. KDIGO 2012 clinical practice guideline for the evaluation and management of chronic kidney disease. Kidney Int. 2013;3:5-14. 
22. Loney PL, Chambers LW, Bennett KJ, Roberts JG, Stratford PW. Critical appraisal of the health research literature prevalence or incidence of a health problem. Chronic Dis Inj Can. 1998;19(4):170-6. PMid:10029513.

23. Passos V, Barreto S, Lima-Costa M. Detection of renal dysfunction based on serum creatinine levels in a Brazilian community: the Bambuí Health and Ageing Study. Braz J Med Biol Res. 2003;36(3):393-401. PMid:12640505. http://dx.doi.org/10.1590/S0100-879X2003000300015.

24. Instituto Brasileiro de Geografia e Estatística. Pesquisa Nacional por Amostra de Domicílio [Internet]. Rio de Janeiro: IBGE; 1998 [citado em 2017 Jul 4]. Disponível em: ftp://ftp.ibge.gov.br/Trabalho_e_Rendimento/ Pesquisa_Nacional_por_Amostra_de_Domicilios_anual/1998/

25. Sociedade Brasileira de Nefrologia. Censo Brasileiro de Diálise: 1999-2015. São Paulo: SBN; 2015 [citado em 2017 Jul 4]. Disponível em: www.sbn.org. br

26. Lessa I. Níveis séricos de creatinina: hipercreatininemia em segmento da população adulta de Salvador, Brasil. Rev Bras Epidemiol. 2004;7(2):17686. http://dx.doi.org/10.1590/S1415-790X2004000200007.

27. Moura L, Prestes IV, Duncan BB, Thome FS, Schmidt MI. Dialysis for end stage renal disease financed through the Brazilian National Health System, 2000 to 2012. BMC Nephrol. 2014;15(1):1. PMid:25008169. http://dx.doi. org/10.1186/1471-2369-15-111.

28. Nascimento MM, Riella M. Raising awareness of chronic kidney disease in a Brazilian urban population. Braz J Med Biol Res. 2009;42(8):750-5. PMid:19649402. http://dx.doi.org/10.1590/S0100-879X2009000800010.

29. Instituto Brasileiro de Geografia e Estatística. Pesquisa Nacional por Amostra de Domicílio [Internet]. Rio de Janeiro: IBGE; 2003 [citado em 2017 Jul 4]. Disponível em: https://ww2.ibge.gov.br/home/estatistica/populacao/ trabalhoerendimento/pnad2003/default.shtm

30. Bastos RMR, Bastos MG, Ribeiro LC, Bastos RV, Teixeira MTB. Prevalence of chronic kidney disease, stages 3, 4 and 5 in adults. Rev Assoc Med Bras. 2009;55(1):40-4. PMid:19360276.

31. Lima AO, Kesrouani S, Gomes RA, Cruz J, Mastroianni-Kirsztajn G. Population screening for chronic kidney disease: a survey involving 38721 Brazilians. Nephrol Dial Transplant. 2012;27(Suppl 3):gfs063. PMid:22498917.

32. Instituto Brasileiro de Geografia e Estatística. Pesquisa Nacional por Amostra de Domicílio [Internet]. Rio de Janeiro: IBGE; 2008 [citado em 2017 Jul 4]. Disponível em: https://ww2.ibge.gov.br/home/estatistica/populacao/ panorama_saude_brasil_2003_2008/default.shtm

33. Barreto SM, Ladeira RM, Duncan BB, Schmidt MI, Lopes AA, Benseñor IM, et al. Chronic kidney disease among adult participants of the ELSABrasil cohort: association with race and socioeconomic position. J Epidemiol Community Health. 2016;70(4):380-9. PMid:26511886. http://dx.doi. org/10.1136/jech-2015-205834.

34. Dutra MC, Uliano EJM, Machado DFGP, Martins T, Schuelter-Trevisol F, Trevisol DJ. Avaliação da função renal em idosos: um estudo de base populacional. J Bras Nefrol. 2014;36(3):297-303. PMid:25317611.

35. Pinho NA, Silva GV, Pierin AMG. Prevalência e fatores associados à doença renal crônica em pacientes internados em um hospital universitário na cidade de São Paulo, SP, Brasil. J Bras Neurol. 2015;37(1):91-7.

36. Schaefer JCF, Pereira MS, Jesus CR, Schuelter-Trevisol F, Trevisol DJ. Estimativa da função renal na população de 18 a 59 anos da cidade de Tubarão-SC: Um estudo de base populacional. J Bras Nefrol. 2015;37(2):18591. PMid:26154638.
37. Pereira ERS, Pereira AC, Andrade GB, Naghettini AV, Pinto FK, Batista SR, et al. Prevalence of chronic renal disease in adults attended by the family health strategy. J Bras Neurol. 2016;38(1):22-30. PMid:27049361. http://dx.doi.org/10.5935/0101-2800.20160005.

38. Instituto Brasileiro de Geografia e Estatística. Pesquisa Nacional Saúde [Internet]. Rio de Janeiro: IBGE; 2013 [citado em 2017 Jul 4]. Disponível em: https://www.pns.icict.fiocruz.br

39. Kirsztajn GM. Avaliação de função renal. J Bras Nefrol. 2009;31(Supl 1):14-20.

40. United States Renal Data System. USRDS Annual Data Report: an Overview of the epidemiology of kidney disease in the United States. Ann Arbor: USRDS Coordinating Center; 2014.

41. Lugon J, Matos JPS. Disparities in end-stage renal disease care in South America. Clin Nephrol. 2010;74(Suppl 1):S66-71. PMid:20979967.

42. Coresh J, Selvin E, Stevens LA, Manzi J, Kusek JW, Eggers P, et al. Prevalence of chronic kidney disease in the United States. JAMA. 2007;298(17):203847. PMid:17986697. http://dx.doi.org/10.1001/jama.298.17.2038.

43. Sesso RC, Lopes AA, Thomé FS, Lugon JR, Martins CT. Inquérito Brasileiro de Diálise Crônica 2014. J Bras Nefrol. 2016;38(1):54-61. PMid:27049365.

44. Consortium CKDP. Association of estimated glomerular filtration rate and albuminuria with all-cause and cardiovascular mortality in general population cohorts: a collaborative meta-analysis. Lancet. 2010;375(9731):2073-81. PMid:20483451. http://dx.doi.org/10.1016/S0140-6736(10)60674-5.

45. Wen CP, Cheng TYD, Tsai MK, Chang YC, Chan HT, Tsai SP, et al. Allcause mortality attributable to chronic kidney disease: a prospective cohort study based on 462293 adults in Taiwan. Lancet. 2008;371(9631):2173-82. PMid:18586172. http://dx.doi.org/10.1016/S0140-6736(08)60952-6.

46. Donfrancesco C, Palleschi S, Palmieri L, Rossi B, Noce CL, Pannozzo F, et al. Estimated glomerular filtration rate, all-cause mortality and cardiovascular diseases incidence in a low risk population: the MATISS study. PLoS One. 2013;8(10):e78475. PMid:24147135. http://dx.doi.org/10.1371/journal. pone. 0078475 .

47. Cusumano AM, Garcia-Garcia G, Gonzalez-Bedat MC, Marinovich S, Lugon J, Poblete-Badal H, et al. Latin American Dialysis and Transplant Registry: 2008 prevalence and incidence of end-stage renal disease and correlation with socioeconomic indexes. Kidney Int Suppl. 2013;3(2):153-6. PMid:25018980. http://dx.doi.org/10.1038/kisup.2013.2.

48. Rosa-Diez G, Gonzalez-Bedat M, Pecoits-Filho R, Marinovich S, Fernandez $S$, Lugon J, et al. Renal replacement therapy in Latin American end-stage renal disease. Clin Kidney J. 2014;7(4):sfu039. PMid:25349696. http:// dx.doi.org/10.1093/ckj/sfu039.

49. Fernandes NM, Chaoubah A, Bastos K, Lopes AA, Divino-Filho JC, Pecoits-Filho R, et al. Geography of peritoneal dialysis in Brazil: analysis of a cohort of 5,819 patients (BRAZPD). J Bras Neurol. 2010;32(3):268-74. PMid:21103690.

50. Lugon J, de Matos J. Disparities in end-stage renal disease care in South America. Clin Nephrol. 2010;74(7 Supl Suppl 1):S66-71. PMid:20979967.

51. Barcellos RC, Matos JP, Kang HC, Rosa ML, Lugon JR. Comparison of serum creatinine levels in different color/race categories in a Brazilian population. Cad Saude Publica. 2015;31(7):1565-9. PMid:26248110. http:// dx.doi.org/10.1590/0102-311X00150814.

52. Bastos MG, Kirsztajn GM. Doença renal crônica: importância do diagnóstico precoce, encaminhamento imediato e abordagem interdisciplinar estruturada 
para melhora do desfecho em pacientes ainda não submetidos à diálise. J Bras Nefrol. 2011;33(1):93-108. PMid:21541469. http://dx.doi.org/10.1590/ S0101-28002011000100013.

53. Barros MBA, Francisco PMSB, Zanchetta LM, César CLG. Tendências das desigualdades sociais e demográficas na prevalência de doenças crônicas no Brasil, PNAD: 2003-2008. Ciênc Saúde Coletiva. 2011;16(9):3755-68.

54. Yamakawa RH, Saito PK, Bedendo J, Silva Jr WV, Carvalho MDB, Borelli SD. Chronic kidney disease: information on southern brazilian patients with kidney disease. Acta Sci Health Sci. 2012;34:247-50. http://dx.doi.org 10.4025/actascihealthsci.v34ispec.16048.

55. Travassos CMR, Viacava F, Laguardia J. Os suplementos saúde na Pesquisa Nacional por Amostra de Domicílios (PNAD) no Brasil. Rev Bras Epidemiol. 2008;11(Supl. 1):98-112. http://dx.doi.org/10.1590/S1415-790X2008000500010.

56. Oliveira MB, Romao JE Jr, Zatz R. End-stage renal disease in Brazil: epidemiology, prevention, and treatment. Kidney Int. 2005;68(97):S82-6. PMid:16014106. http://dx.doi.org/10.1111/j.1523-1755.2005.09714.x.

57. Barros MBA, Francisco PMSB, Zanchetta LM, César CLG. Tendências das desigualdades sociais e demográficas na prevalência de doenças crônicas no Brasil, PNAD: 2003-2008. Ciênc Saúde Coletiva. 2011;16(9):3755-68. http://dx.doi.org/10.1590/S1413-81232011001000012 .

58. Peres LAB, Biela R, Hermann M, Matsuo T, Ann HK, Camargo MT, et al. Estudo epidemiológico da doença renal crônica terminal no Oeste do Paraná: uma experiência de 878 casos atendidos em 25 anos. J Bras Nefrol. 2010;32(1):51-6. http://dx.doi.org/10.1590/S0101-28002010000100010.

59. Pestana JOM, Galante NZ, Tedesco-Silva H Jr, Harada KM, Garcia VD, Abbud-Filho M, et al. O contexto do transplante renal no Brasil e sua disparidade geográfica. J Bras Nefrol. 2011;33(4):472-84. http://dx.doi. org/10.1590/S0101-28002011000400014.
60. Sesso RC, Lopes AA, Thomé FS, Lugon JR, Watanabe Y, Santos DR. Diálise crônica no Brasil-relatório do censo brasileiro de diálise, 2011. J Bras Nefrol. 2012;34(3):272-7. PMid:23099833.

61. Szuster DAC, Caiaffa WT, Andrade EIG, Acurcio FA, Cherchiglia ML. Sobrevida de pacientes em diálise no SUS no Brasil Survival analysis of dialysis patients in the Brazilian Unified National Health System. Cad Saude Publica. 2012;28(3):415-24. PMid:22415174. http://dx.doi.org/10.1590/ S0102-311X2012000300002.

62. Cunha CB, León ACP, Schramm JMA, Carvalho MS, Souza PRB Jr, Chain R. Tempo até o transplante e sobrevida em pacientes com insuficiência renal crônica no Estado do Rio de Janeiro, Brasil, 1998-2002 Time to kidney transplantation in chronic renal. Cad Saude Publica. 2007;23(4):805-13. PMid:17435878. http://dx.doi.org/10.1590/S0102-311X2007000400008.

63. Gerschman S, Santos MAB. O Sistema Único de Saúde como desdobramento das políticas de saúde do século XX. Rev Bras Cienc Soc. 2006;21(61):177227. http://dx.doi.org/10.1590/S0102-69092006000200010.

64. Go AS, Chertow GM, Fan D, McCulloch CE, Hsu CY. Chronic kidney disease and the risks of death, cardiovascular events, and hospitalization. N Engl J Med. 2004;351(13):1296-305. PMid:15385656. http://dx.doi. org/10.1056/NEJMoa041031.

65. Higgins JP, Green S. Cochrane handbook for systematic reviews of interventions. Hoboken: Wiley Online Library; 2008.

66. Akers J, Aguiar-Ibáñez R, Baba-Akbari Sari A. CRD’s guidance for undertaking reviews in health care. New York: Centre for Reviews and Dissemination; 2009.

Recebido em: Jul. 04, 2017 Aprovado em: Set. 18, 2017 